\title{
Laser cooling of rubidium atoms in a 2D optical lattice
}

\section{Chunhua Wei \& Carlos C. N. Kuhn}

To cite this article: Chunhua Wei \& Carlos C. N. Kuhn (2018) Laser cooling of rubidium atoms in a 2D optical lattice, Journal of Modern Optics, 65:10, 1226-1234, DOI:

10.1080/09500340.2018.1429684

To link to this article: https://doi.org/10.1080/09500340.2018.1429684

\section{Published online: 02 Feb 2018.}

Submit your article to this journal $\pi$

III Article views: 147

View Crossmark data $₫$ 


\title{
Laser cooling of rubidium atoms in a 2D optical lattice
}

\author{
Chunhua Wei, ${ }^{a, b}$ and Carlos C. N. Kuhn ${ }^{c}$ \\ aDepartment of Instrument Science and Technology, College of Mechatronic Engineering and Automation, National University of Defense \\ Technology, Changsha, China; ${ }^{b}$ Quantum Sensors Lab, Department of Quantum Science, Australian National University, Canberra, Australia; \\ ${ }^{c}$ Center for Quantum and Optical Science, Swinburne University of Technology, Melbourne, Australia
}

ABSTRACT

Lossless polarization gradient cooling of $4 \times 10^{7}{ }^{87} \mathrm{Rb}$ atoms in a far-detuned 2D optical lattice is demonstrated. Temperatures down to $1 \mu \mathrm{K}$ and phase space densities as high as $1 / 1000$ are achieved in a total duty cycle of $215 \mathrm{~ms}$. It is shown that utilizing the vector component of the optical lattice allows lower temperatures to be achieved when compared with pure scalar lattices.

\section{ARTICLE HISTORY}

Received 13 September 2017

Accepted 8 January 2018

\section{KEYWORDS}

Laser cooling; optical lattice; polarization gradient cooling

\section{Introduction}

Laser cooling techniques which can produce low temperatures at high densities are of substantial practical importance. Numerous technologies benefit from a bright atomic source, including atomic clocks (1-3), atom interferometers (4-6), quantum computing (7-9), quantum communication (10) and the production and use of BoseEinstein condensates (BECs) $(11,12)$.

The most commonly used method to trap and cool neutral atoms is the magneto-optical trap (MOT), a configuration of red detuned lasers and a quadrupole magnetic field (13). The MOT is designed to harness the Doppler effect to cool a large number of atoms to the Doppler limit, $T_{\text {Doppler }}=\hbar \gamma / 2 k_{B}$, where $\gamma$ is the laser cooling transition linewidth (typically around $2 \pi \times 10$ $\mathrm{MHz}$ for alkali atoms) and $k_{B}$ is the Boltzmann constant, with temperatures around $250 \mu \mathrm{K}$ predicted (for $\mathrm{Rb}$ atoms where the transition linewidth is $2 \pi \times 6.06 \mathrm{MHz}$ the $\left.T_{\text {Doppler }} \approx 146 \mu \mathrm{K}\right)$. Experimental application of the MOT was found to cool atoms significantly below this limit, through a process termed Sisyphus cooling. This process arises through a combination of optical pumping and the polarization gradients experienced by the atoms moving through the optical field of the MOT beams, allowing significantly more energy to be extracted from the laser cooled atoms and leading to temperatures over an order of magnitude less than Doppler cooling (14). This technique became known as polarization Gradient Cooling (PGC).

Polarization gradient cooling was a result spurred by a push to create cooling schemes approaching the recoil limit, $T_{\text {recoil }}=(\hbar k)^{2} / 2 m k_{B}\left(361.96 \mathrm{nK}\right.$, for ${ }^{87} \mathrm{Rb}$ atoms $)$, where $k$ is the wavenumber of the cooling light and $m$ is the mass of the atom. A key insight was to realize that imposing an optical lattice could engineer dark states that led to temperatures an order of magnitude lower than standard PGC, with $1.1 \mu \mathrm{K}$ demonstrated for Cs $(15,16)$. The use of optical lattice traps as aids to adiabatic cooling of Cs have also been verified, with temperatures around $700 \mathrm{nK}$ achieved in 3D lattices (17). The pinnacle of laser cooling method while keeping atom number constant during the cooling process is 3D Raman sideband cooling (and derivatives) in far-detuned optical lattices, which can greatly increase the ground-state occupation fraction of lattice-bound atoms and produce temperatures as low as $290 \mathrm{nK}(18-20)$ that technique create a fictitious cooling transition with a very narrow linewidth that decrease the recoil limit. It should be noted that significant progress on low-temperature, high-phase space density laser cooling has been achieved using Doppler cooling on narrow forbidden lines (21-23), leading to Bose-Einstein condensation in Sr using laser cooling only (24). Ultimately, the techniques of laser cooling aim to engineer a narrow line absorption line in any atomic species.

We demonstrate polarization gradient cooling in a fardetuned 2D optical lattice, applied to efficiently cool a sample of ${ }^{87} \mathrm{Rb}$ atoms. The configuration is shown to achieve temperatures of $1 \mu \mathrm{K}$ in two dimensions and is compared with optimized free space PGC in the same system, demonstrating an order of magnitude improvement. $4 \times 10^{7}{ }^{87} \mathrm{Rb}$ atoms are trapped in the lattice, resulting in a phase space density as high as $1 / 1000$ at an atomic density of $3 \times 10^{11} \mathrm{~cm}^{-3}$. The recoil energy, $E_{r}=\hbar^{2} k^{2} / 2 m$, is used to compare the cooling efficiency 


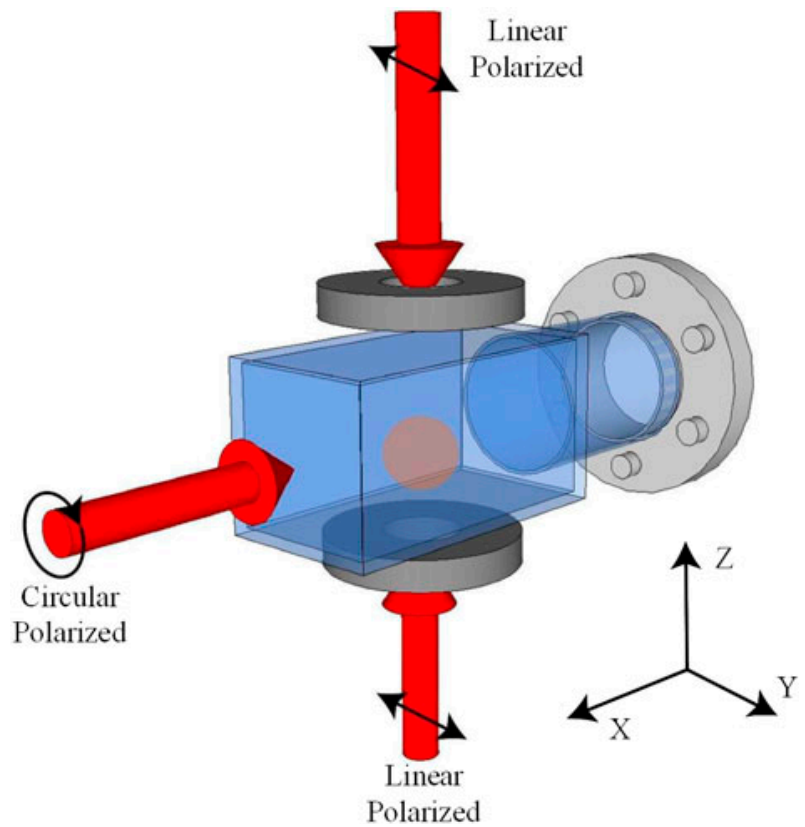

Figure 1. Schematic view of the lattice beams configuration on the MOT Cell. The vertical beams are linear polarized along the $y$ direction from the $\pm z$-axis and the horizontal beam is right-handed circular polarized along $x$ direction. The coils in the figure generate a quadrupole magnetic field (anti-Helmholtz configuration to be used in the MOT). During the lattice and PGC the magnetic field is turned off, no current is supplied for the coils.

between differing atomic species, with the results achieving approximately $22 E_{r}$ in ${ }^{87} \mathrm{Rb}$, comparable to leading results in ${ }^{133} \mathrm{Cs}$ that reaches temperatures around $700 \mathrm{nk}$ $\left(28 E_{r}\right)(17)$. A schematic of the vector lattice cooling system is shown in Figure 1, which is simple and easy to be implemented, requiring only three additional laser beams beyond a standard MOT setup. Compared with a scalar lattice, this configuration (i) minimizes heating due to re-absorption of cooling photons and (ii) generates an exchange population from bright to dark states due to a non-degenerate potential similar to PGC.

\section{Theoretical background}

In this section, the theoretical background on optical dipole potential is briefly reviewed to help the comparison between the two optical lattices presented in this work. Let's consider three laser beams with frequency $\omega_{L}$, two of which propagate along the $z$ direction with the final along $x$ direction. The total electric field is then given by

$$
\mathbf{E}(x, y, z, t)=\mathbf{E}_{0}(x, y, z) e^{-i \omega_{L} t}+\text { c.c. },
$$

where

$$
\mathbf{E}_{0}(x, y, z)=E_{01} \hat{\mathbf{e}}_{1} e^{i k z}+E_{02} \hat{\mathbf{e}}_{2} e^{-i k z}+E_{03} \hat{\mathbf{e}}_{3} e^{-i k x}
$$
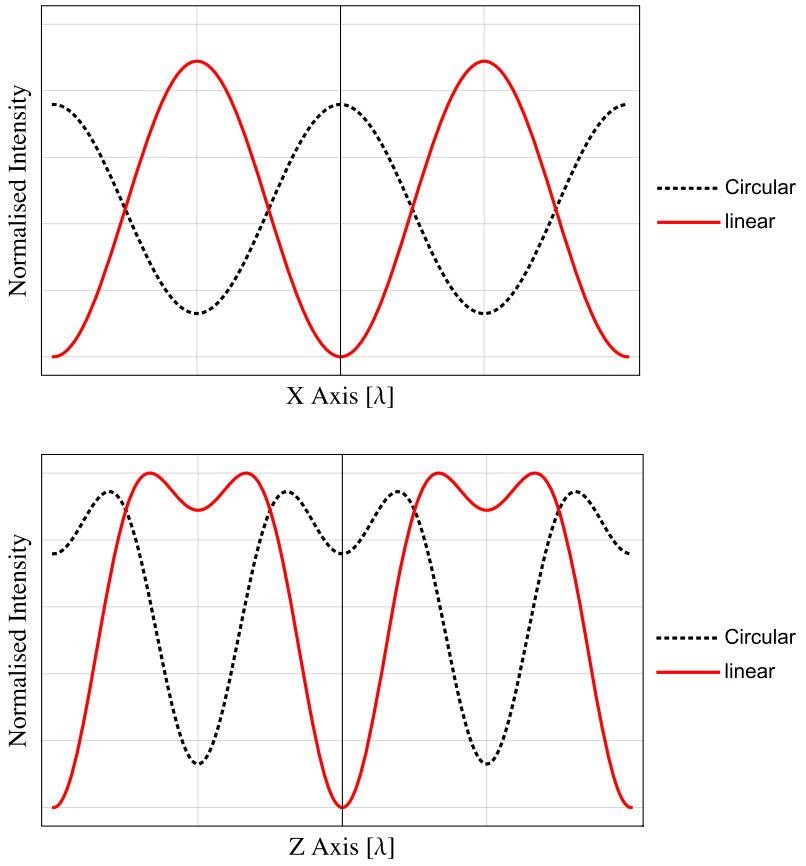

Figure 2. Normalized Intensity along the $x$ - and $z$-axis, obtained from Equation (5), $I \propto\left|\mathbf{E}_{\text {lin }}\right|^{2}$ (red solid line), and from Equation (7), I $\propto\left|\mathbf{E}_{\text {cir }}\right|^{2}$ (black dashed curve).

describes the amplitude of the electric field given $E_{0 i}$ are the amplitudes of the electric field for each of the three laser beams, $\hat{\mathbf{e}}_{i}$ are their local unit polarization vector, $k=2 \pi / \lambda$ is the wavenumber and $\lambda$ is the wavelength of light. In the case of multi-level atoms and accounting for linear and circular light, the optical dipole potential can be expressed by (25)

$$
U(\mathbf{r})=\frac{\pi c^{2} \Gamma}{2 \omega_{0}^{3}}\left(\frac{2+P g_{F} m_{F}}{\Delta_{2, F}}+\frac{1-P g_{F} m_{F}}{\Delta_{1, F}}\right) I(\mathbf{r}) .
$$

where $I(\mathbf{r})=2 \epsilon_{0} c\left|E_{0}(x, y, z)\right|^{2}$ is the lattice light intensity, $\omega_{0}$ is the atomic resonance frequency, $g_{F}$ is the Landé factor and $m_{F}$ is the magnetic quantum number for the total angular moment $F$. Here $P$ stands to the laser polarization $(P=0$ for linearly polarized light, $P= \pm 1$ for circularly $\sigma^{ \pm}$polarized light). The detunings $\Delta_{1, F}$ and $\Delta_{2, F}$ are calculated in such way the total dipole potential takes into account the $D_{1}$ and $D_{2}$ line, respectively, (see (25) for further details). In the case of large detunings, the dipole potential can be linearly expanded in term of $\Delta_{F S}^{\prime} / \Delta$, where we defined the detuning from the centre of the $D$-line doublet $\Delta$ and the $\Delta_{F S}^{\prime}$ is the excited fine structure splitting. Thus the optical dipole potential becomes

$$
U(\mathbf{r})=\frac{3 \pi c^{2} \Gamma}{2 \omega_{0}^{3} \Delta}\left(1+\frac{1}{3} P g_{F} m_{F} \frac{\Delta_{F S}^{\prime}}{\Delta}\right) I(\mathbf{r}) .
$$




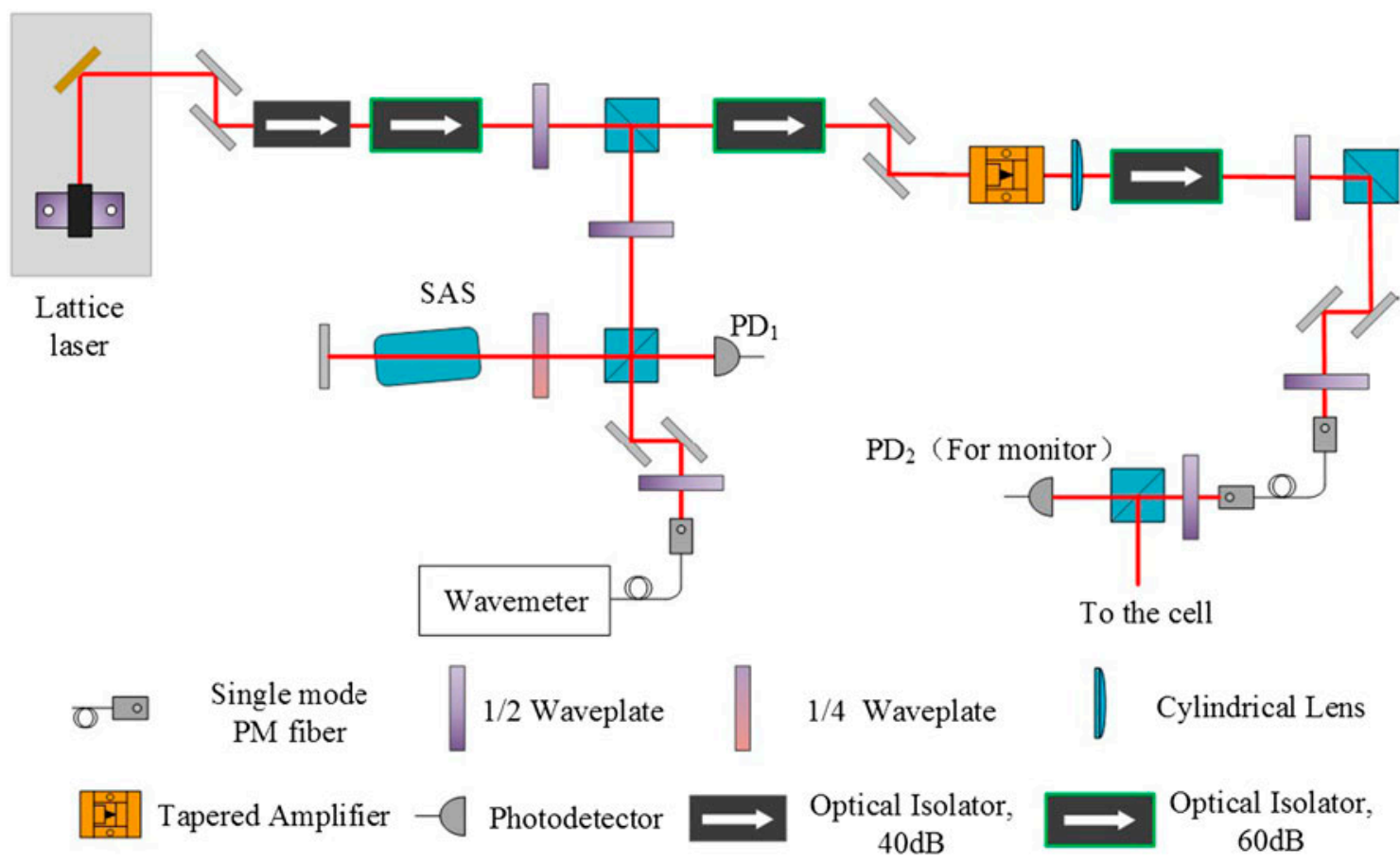

Figure 3. Laser setup for the optical lattice. An ECDL is used to seed a tapered amplifier on the science table. This light undergoes spatial filtering before the cell, with the laser frequency monitored using a wavemeter.

It becomes clear from Equation (4) that in the case of linear polarized light $(P=0)$, it recovers the optical dipole potential calculated when using the well-known rotating wave approximation.

In this work, where $\Delta_{F S}^{\prime}>\Delta_{1, F}, \Delta_{2, F}$ and $\Delta_{1, F}>>$ $\Delta_{2, F}$ with unresolved excited-state hyperfine splitting, the Equation (3) remain valid. It is important to notice that in this case, the polarization of the light plays a stronger role. The circularly polarized light lifts the degeneracy of the magnetic hyperfine states of the ground states, and it can be understood as an 'effective magnetic field' (26).

In order to understand the oscillatory response of potential and its spatial polarization dependency, Equation (2) need to be taken a close look. Two cases are compared here, the pure scalar lattice and the vectorial lattice. For the first configuration, there have $E_{01}=E_{02}=E_{03}=E_{0}$ and $\hat{\mathbf{e}}_{i}=\hat{\mathbf{e}}_{y}$ for $i=\{1,2,3\}$, where $\hat{\mathbf{e}}_{y}=[0,1,0]$ is the unitary Cartesian vector. Setting those parameters in Equation (2), we get

$$
\mathbf{E}_{\text {lin }}=E_{0} \hat{\mathbf{e}}_{y}[\cos (k x)+2 \cos (k z)-i \sin (k x)] .
$$

For the second configuration, Figure 1, the beam along the $x$-axis is circularly polarized, such that

$$
\hat{\mathbf{e}}_{3}=-\frac{\hat{\mathbf{e}}_{y}+i \hat{\mathbf{e}}_{z}}{\sqrt{2}},
$$

This specific configuration generates a lattice similar to the lin $\perp$ lin configuration described in (27). Equations (6) and (2) leads to

$$
\begin{array}{r}
\left.\mathbf{E}_{c i r}=-\frac{E_{0}}{\sqrt{2}[}(\cos (k x)-2 \sqrt{2} \cos (k z)) \hat{\mathbf{e}}_{y}-\sin (k x) \hat{\mathbf{e}}_{z}\right] \\
+i \frac{E_{0}}{\sqrt{2}}\left[\sin (k x) \hat{\mathbf{e}}_{y}-\cos (k x) \hat{\mathbf{e}}_{z}\right]
\end{array}
$$

Figure 2 shows the normalized intensity obtained for both configurations using Equations (5) and (7), $I \propto|\mathbf{E}|^{2}$. The solid red line and dashed black curve for linearly and circularly polarized light for the third lattice beam, respectively. No periodicity is observed in the $y$ direction. Here it is clear that the two configuration generates a very similar scalar component for the light shift.

Equation (7) outlines the spatial dependence of the polarization of the light in this configuration. It can be seen that the polarization of the light varies spatially from pure $\sigma^{-}$at $z=\lambda / 8$ and $z=7 \lambda / 8$ to pure $\sigma^{+}$at $z=\lambda / 4$ and $z=3 \lambda / 4$ for $x=0$ relative to the $x$ direction. In contrast, along the $x$-axis at $z=0$, the light is never purely circularly polarized, but a linear combination of both $\sigma$ polarization forming a periodic potential, Figure 2. This periodic potential results in a tensor component of the light shift experienced by the atoms and behaves like a first-order Zeeman shift, breaking the degeneracy of the lattice, as mentioned before. 


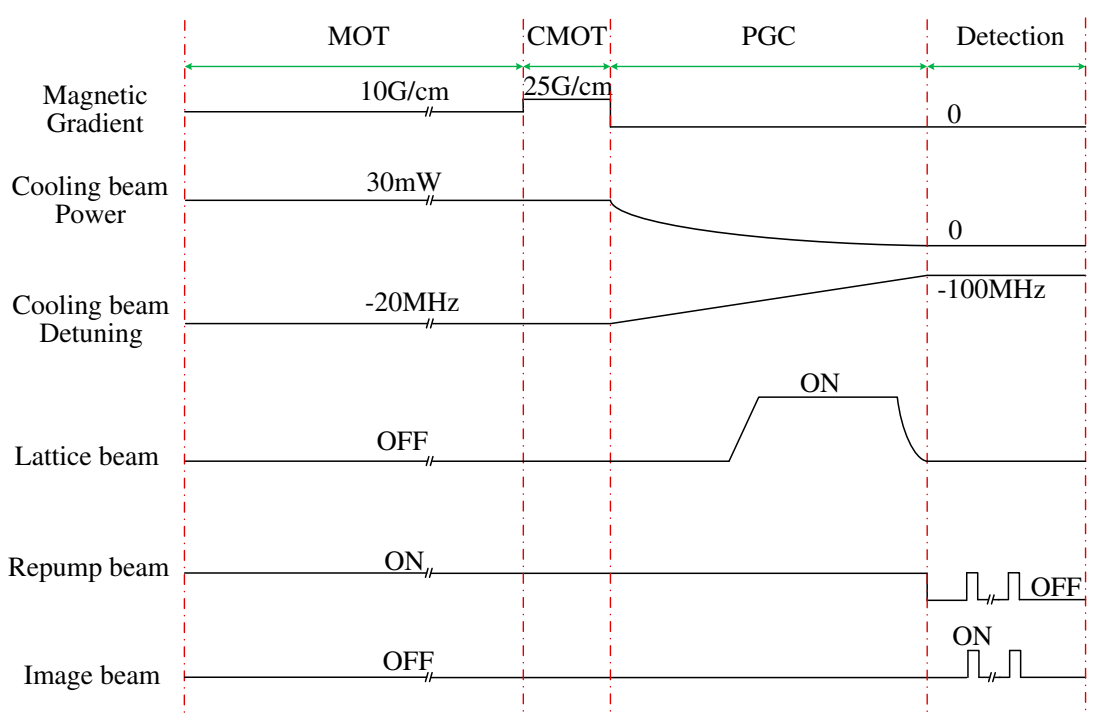

Figure 4. The diagram of cooling experimental sequence. The lattice beam TTL is off when implementing a standard PGC process.

Similar schemes have been studied theoretically with respect to 2D Sisyphus cooling (28). Basically, each of the Zeeman sublevels see a different potential resulting from the lattice.

We demonstrate that the two configuration creates a periodic potential along the $x-$ and $z$-axis. The main observed difference between the two schemes is that when using circular light in the third beam, the optical dipole potential is not the same for all the $m_{F}$ states due to the fact the polarization of the light changes as a function of position. This difference is a strong candidate to be the reason for the improvement in the cooling. Although the physics behind needs further investigation.

\section{Experimental setup}

\subsection{Laser setup}

The experiment utilizes external cavity diode lasers (ECDL) locked to an atomic transition using saturated absorption spectroscopy (SAS). The $40 \mathrm{~mW}$ trapping laser (red detuned from the $F=2 \rightarrow F^{\prime}=3$ of the ${ }^{87} \mathrm{Rb}$ $\mathrm{D}_{2}$ transition hyperfine level) is amplified $(\sim 700 \mathrm{~mW})$ then sent through an acoustic-optic modulator using a double-pass setup (DP AOM). The laser mode is then cleaned through $3 \mathrm{~m}$ of polarization maintaining singlemode (PMSM) fibre, resulting in about $240 \mathrm{~mW}$ of trapping light sent to the glass cell. Immediately after the locking loop, a small amount of light is removed for use as a probe beam in an absorption image system and is capable of being tuned independently of the trapping light using an AOM double-pass set up. The image beam, in the science table, is located horizontally and orthogonal to the horizontal lattice beam. The $60 \mathrm{~mW}$ repump laser $\left(F=1 \rightarrow F^{\prime}=2\right)$ is passed through an AOM, with the first-order diffracted light fibre coupled to the glass cell resulting in a total power of $12 \mathrm{~mW}$ at the science cell.

The geometric setup to generate the optical lattice potential is shown in Figure 3. The ECDL used to generate the lattice is amplified and spatially filtered through $3 \mathrm{~m}$ of PMSM fibre. A home built wavemeter is added to accurately detune the laser from the $D_{2}$ transition (29). The laser can be locked at any frequency using a software implementation of a proportional integral derivative (PID) controller and is experimentally simple to implement, requiring only $10 \mu \mathrm{W}$ of light coupled into the wavemeter fibre from the back of the Saturated Absorption Spectroscopy (SAS) beam splitter. In order to avoid spontaneous emission heating of the cold atoms, the optical lattice is typically set to be $20 \mathrm{GHz}$ detuned to the red of the $F=2 \rightarrow F^{\prime}=3$ transition.

The intensity of the lattice is directly controlled by pulsing the current through the tapered amplifier using a function generator (Agilent 33250A) and a Wavelength Electronics PLD5000 5A laser diode driver. This setup is useful for producing a high-power output from a tapered amplifier when there is limited seed light available, or when the application only requires a large amount of power for a short amount of time (30). A polarizing beam splitter (PBS) is used to divide the total lattice power. Waveplates are used prior to input to the glass cell to adjust the polarization of each lattice beam. Three beams are used to build a $2 \mathrm{D}$ lattice. The vertical lattice is linearly polarized, with a $1 / e^{2}$ waist of $5 \mathrm{~mm}$, created by retro-reflecting a single input beam. The third and single horizontal lattice beam can be circular or linearly polarized. When the horizontal lattice beam is linearly polarized a pure scalar 2D lattice is formed. On the other 
(a)
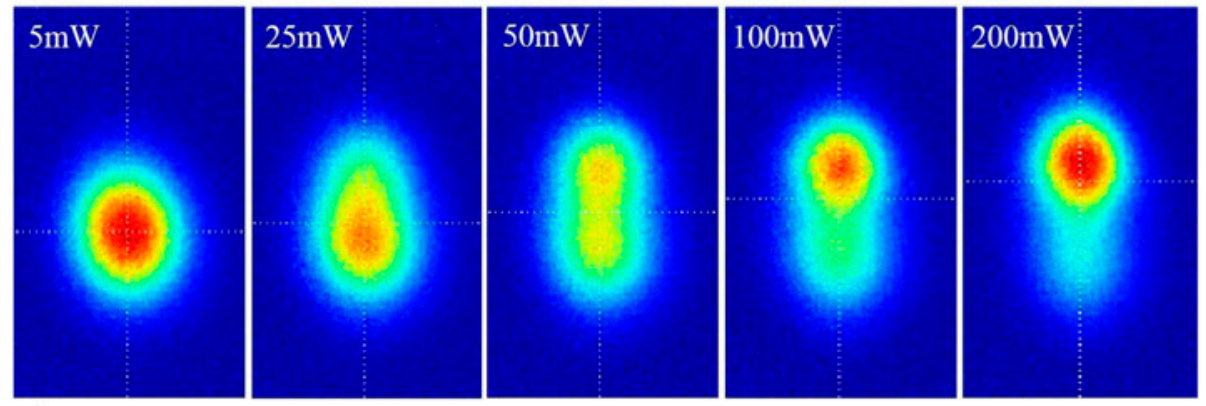

(b)
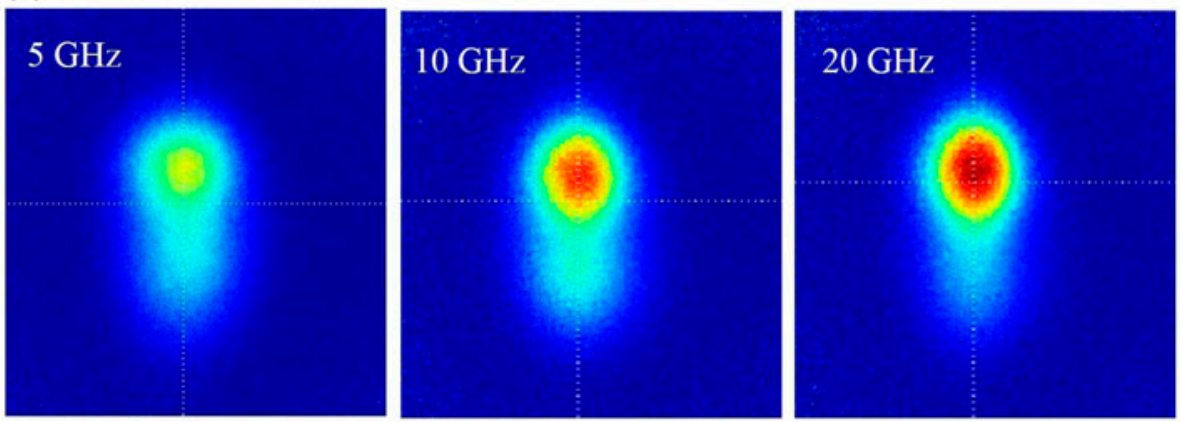

Figure 5. (a) Adiabatically loading of the optical lattice at $20 \mathrm{GHz}$ detuning to the red from the $F=2 \rightarrow F^{\prime}=3$ transition. Atoms are loaded adiabatically using a $3 \mathrm{~ms}$ linear ramp and held in the lattice for $10 \mathrm{~ms}$. The atoms are imaged $15 \mathrm{~ms}$ after release by absorption image technique using a probe beam at resonance with the atomic colling transition. The first picture shows the cloud is not affected by the lattice beam. As the lattice intensity increases, it can hold atoms to against the gravity because of the trap depth increase. So, more atoms can load into the lattice, and fewer atoms fall. The trapped and untrapped atoms have an offset of around $15 \mathrm{~ms}$ in the TOF image. (b) The lattice is switched on according to the above protocol at $200 \mathrm{~mW}$ at different detuning. Tuning closer to resonance at this power results in a clear loss of atoms due to heating.

hand, a circularly polarized beam generates a 2D lattice with a vector gradient for the atoms in two perpendicular directions.

\subsection{Experimental sequence}

The experimental sequence starts with $4 \times 10^{7}$ of ${ }^{87} \mathrm{Rb}$ atoms captured in $200 \mathrm{~ms}$ in a standard 3D MOT (13) configuration. The trapping light with total power of $240 \mathrm{~mW}$ is divided into three counterpropagating beams using beam splitters and waveplates. The light polarizations (sigma plus and sigma minus for each counterpropagating beam respectively) are adjusted using quarterwave plates just before reach to glass cell. This configuration provides a damping and restoring force in the atoms when combined with the magnetic quadrupole potential generated from a pair of coils on the anti-Helmholtz configuration.

Just before the PGC, an initial stage of MOT compression is implemented by increasing the magnetic field gradient from 10 to $25 \mathrm{G} / \mathrm{cm}$. During our PGC, the magnetic field is then switched off (Figure 4) in $100 \mu$ s by turning off the power supplier that generates current for the coils and the cooling beam are swiped from -20 to $-100 \mathrm{MHz}$ detuned from the cooling transition during
$15 \mathrm{~ms}$. Although the intensity of the light is not actively varied during our PGC, it does decrease due to variation in the coupling efficiency of the Acoustic-Optic Modulator $(\mathrm{AOM})$ and the optical fibre generating due to change in the frequency of the light which generates a smooth decay in intensity. The intensity of the repump light is linearly ramped down to 0 during this phase, to limit the population in the $F=2$ ground state then decreasing the heating effect due to spontaneous emission from the cooling light. At the end of this stage, there are $4 \times 10^{7}$ ${ }^{87} \mathrm{Rb}$ atoms in the $F=1$ state with a $3 \mathrm{D}$ temperature of approximately $12 \mu \mathrm{K}$.

In order to achieve further cooling, the atoms are adiabatically loaded into the vectorial $2 \mathrm{D}$ optical lattice during the end of the PGC stage, the optimization of the intensity and detuning of the lattice light to load more atoms and do not heat up the cloud is demonstrated in Figure 5.

As detailed in the following sections, the presence of the lattice potential during the PGC minimizes heating effects due to spontaneous emission. The lattice also breaks the degeneracy of the Zeeman sub-levels allowing further extraction of energy by generating a fictitious magnetic field parallel to the k-vector of the light $(26,31-33)$. The cloud is adiabatically released from the lattice when the 


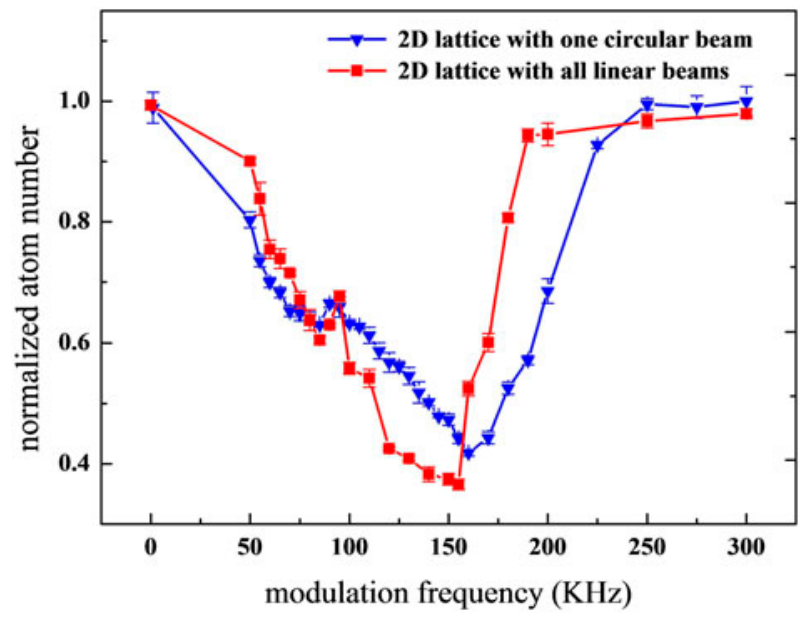

Figure 6. Vibration frequency of the 2D optical lattice for the two different configurations, obtained by modulating the trap intensity at a variable frequency. The amplitude of the modulation is $30 \mathrm{~mW}$. Resonant parametric heating and consequent loss of atoms from the trap is observed when this frequency coincides with twice the trap frequency in the tightly confining direction of the lattice. The two configurations correspond to the 2D circularly polarized lattice (blue triangles) and the 2D linearly polarized lattice (red square) and form similar scalar lattice potential with same trap frequency.

MOT beams are extinguished. With this sequence, the atoms are cooled to temperatures around $1 \mu \mathrm{K}$ in two dimensions, with no observable atom loss.

\section{Results and discussion}

In order to narrow down all the possible reasons for the cooling observed in this work, the atoms must be adiabatically loaded and unloaded from the lattice. The heating may be minimized by altering the intensity of the light according to

$$
I(t)=\frac{I(0)}{\left(1+\Gamma_{A} t\right)^{2}}
$$

where $I(0)$ is the initial intensity, and $\Gamma_{A}$ is the time scale for adiabatic unloading (17).

The time scale of loading and unloading the lattice was examined through measurements of temperatures of the cloud before the load and after to unload. The temperature measurement is done by time-of-flight (TOF) expansion (34). The width of a thermal cloud will ballistic expand during TOF following the equation $\sigma(t)^{2}=\sigma_{0}^{2}+$ $\left(k_{B} T / m\right) t^{2}$, where $\sigma_{0}$ is the Gaussian radius of the cloud in the trap. The temperature is estimated by letting the cloud expand and measuring the width of the cloud by fitting the density distribution profile of the cloud to a Gaussian distribution for different TOF. The profiles are obtained by absorption imaging technique.
We experimentally determined the loading and unloading time by turning the lattice beams at the conclusion of the PGC stage and holding the atoms for $10 \mathrm{~ms}$, allowing the not trapped atoms to fall. The lattice beam then is turned off using the Equation (8) releasing the atoms for the temperature measurements. The optimum time scale of loading (performed using a linear ramp) and unloading was $3 \mathrm{~ms}$ and $1.5 \mathrm{~ms}$ respectively, optimized to ensure the process produced no extra heating or cooling of the atom cloud due to adiabatic expansion (17).

The optimization of the lattice detuning and intensity is demonstrated in Figure 5. For the figure, the atoms are held in the lattice (detuned $20 \mathrm{GHz}$ to the red of $F=2 \rightarrow$ $F^{\prime}=3$ ) for $10 \mathrm{~ms}$ after the MOT light is extinguished, and then allowed to expand for $15 \mathrm{~ms}$ before they are imaged at resonance. The figure shows that, in this regime, as the intensity of the lattice is increased it can hold more atoms. The loading and heating of the atoms are much more sensitive to the detuning than to the intensity. This is because of the scattering rate is $\propto I / \delta^{2}$, while the trap depth is $\propto I / \delta$. The difference is shown very clearly in Figure 5(b), where using the same lattice protocols the lattice power is held constant at $200 \mathrm{~mW}$ and the lattice detuning is varied. We can see an increase in the number of trapped atoms at greater detuning, despite the fact the potential depth is smaller, because of heating due to the lattice laser is decreased.

In order to compare the scalar and vector lattice configurations, the polarization of the horizontal lattice beam changes from circular to linear. The trap frequencies of both lattices were measured by modulating the lattice potential, resulting in resonant heating of the atoms when the modulation frequency is twice the oscillation frequency. For strong heating, a readily detectable loss of atoms from the trap will result. Figure 6 shows the remain trapped atoms as a function of the applied modulation frequency. The maximum atom loss (valley in the plot) is observed for both configurations at $\sim 160 \mathrm{kHz}$, implying a similar lattice depth for both schemes. These data assert that the observed cooling is not due to different potential depths between the lattices, and is, therefore, the cooling observed in this work is not only due to the minimization of the re-absorption of scattered light during the PGC as demonstrated in $(17,35)$.

Cooling in the scalar and vector lattices were also examined and compared. In the scalar configuration, the atoms will see only linearly polarized light along the $y$-axis generating a periodic potential along the $x$ and $z$ axes. In this setup, the light shift is purely due to the scalar component of the field and equal for all $m_{F}$ states. The cooling observed in this configuration is due to the deep lattice which decreases the problem of heating due to re-absorption of spontaneously emitted 
(a)

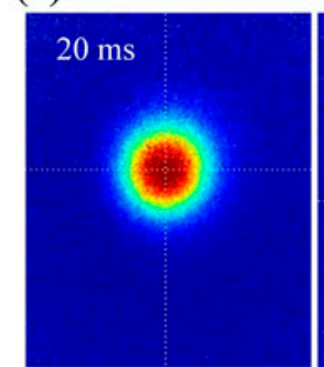

(b)

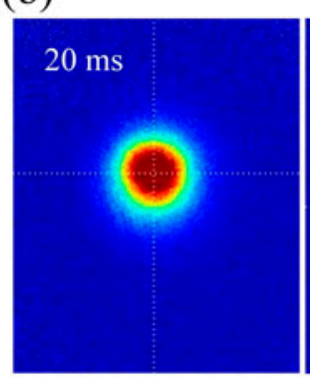

(c)

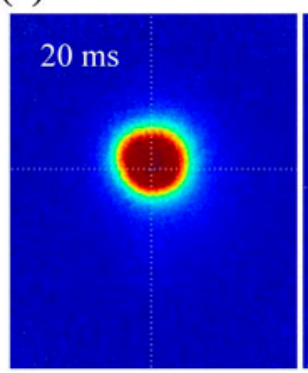

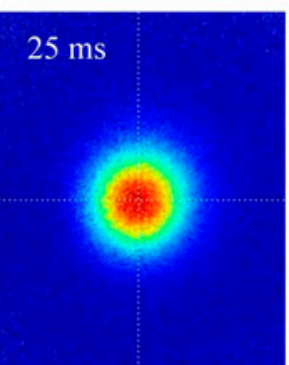
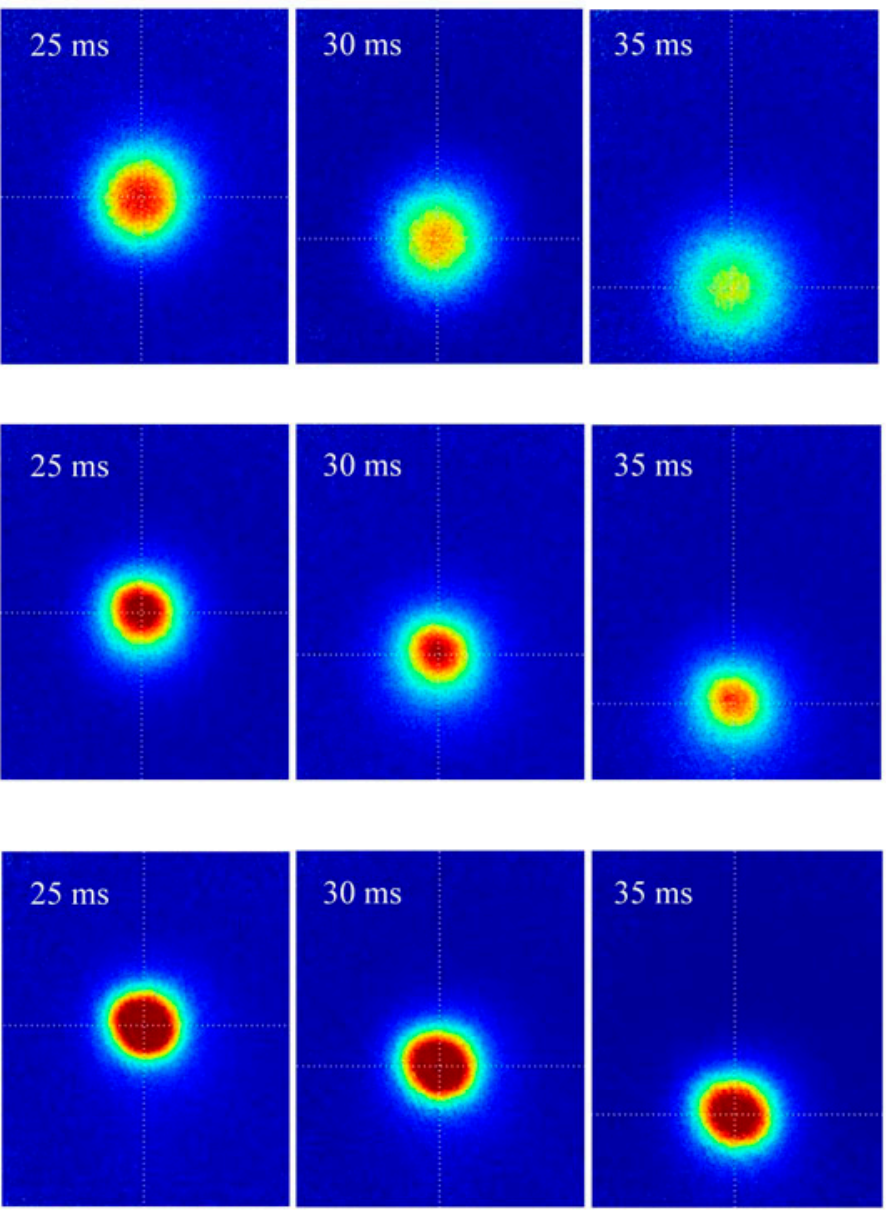

Figure 7. Time-of-flight measurement of the atomic cloud for 20, 25, 30 and $35 \mathrm{~ms}$ for (a) the free space PGC process, (b) PGC in a deep scalar 2D optical lattice, formed using three linear polarized laser beams, and (c) PCG in the vector field 2D optical lattice, formed using two linear polarized beams with a third circular polarized beam. The pictures are in the $x-z$ plane and the lattice is on for $4 \mathrm{~ms}$.

photons from the cooling light (35). In other words, we consider the cooling in this configuration is caused by the increase in the mean free path of the scattered atoms by decreasing the atom density per site and an inhomogeneous Stark shift detuning the scattered photons from resonance. Using this method, it is possible to obtain samples of ${ }^{87} \mathrm{Rb}$ atoms at temperatures of $\sim 6 \mu \mathrm{K}$ in two dimensions. The temperatures are measured using a Gaussian velocity distribution fit to multiple time-offlight (TOF) absorption images. Figure 7(b) shows the corresponding absorption images of a cloud adiabatically released from the scalar 2D optical lattice at varying timeof-flight expansions. Figure 7(b) clearly shows improvement in the cooling when compared with the free space PGC in Figure 7(a).

Tuning the horizontal laser lattice beam to be circularly polarized light, as in Equation (6), the 2D lattice will generate a vector gradient for the atoms. Unlike the previous case, this configuration creates a Sisyphus potential in addition to the periodic potential, producing a gradient force acting on the atoms, and hence cooling to lower temperatures.

Using this vector lattice, $4 \times 10^{7}$ atoms of ${ }^{87} \mathrm{Rb}$ were cooled in two dimensions to temperatures of $1.62 \pm$ $0.38 \mu \mathrm{K}, 1.2 \pm 0.34 \mu \mathrm{K}$, respectively, with the entire cooling scheme taking less than $250 \mathrm{~ms}$, including the MOT loading. The peak phase space density was measured to be $\sim 1 \times 10^{-3}$. Figure 7 (c) shows the TOF absorption images of the cloud released from the vector optical lattice. Here it is very clear that the cloud is much cooler than Figure 7 (a) and (b). The intensity of lattice beam dependence of the cooling is shown in Figure 8 with the sample reaching a minimum temperature for moderate laser power when red detuned $20 \mathrm{GHz}$ from resonance. An extension to $3 \mathrm{D}$ cooling in a vector lattice was not experimentally investigated in this work due to a lack of laser power. Although a simple extension of the model, presented in the theoretical section, suggests that is possible to build a $3 \mathrm{D}$ vectorial lattice by adding a 4 th beam propagating along the $y$-axis with circularly polarized light. 


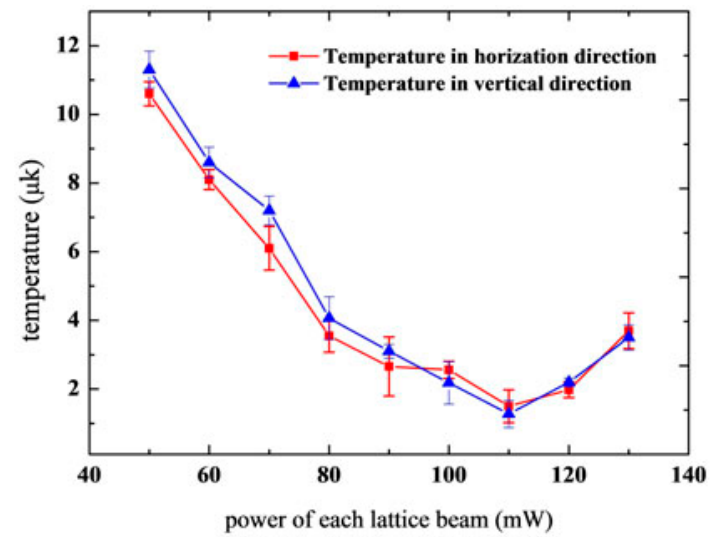

Figure 8. Temperature in the $x$ and $z$ directions as a function of the lattice laser power at $20 \mathrm{GHz}$ detuned.

\section{Conclusion}

A vector optical lattice was investigated for cooling, with the configuration combining deep lattice and Sisyphus cooling. The vector gradient generated by the $2 \mathrm{D}$ lattice allows lossless cooling to temperatures around $1 \mu \mathrm{K}$ in two dimensions. The ability to achieve low-temperature and high-phase space density in a short time scale unlock the prospect of such a system being used in highbandwidth precision measurement applications. This cooling system could also be used as a pre-cooling stage before evaporative cooling to Bose-Einstein condensation.

\section{Acknowledgements}

The authors gratefully acknowledge the support of the Australian Research Council Discovery program. The authors especially thank Assoc. Prof. Nicholas Robins and Prof. John Close for the help, mentoring, and laboratory space provided. The authors also thank the Atomlaser and Quantum Sensor group for productive discussions on the subject.

\section{Disclosure statement}

No potential conflict of interest was reported by the authors.

\section{Funding}

This work was supported by Australian Research Council Discovery program [grant number DP140101779], [grant number DP160104965].

\section{References}

(1) Gibble, K.; Chu, S. Laser-cooled Cs Frequency Standard and a Measurement of the Frequency Shift Due to Ultracold Collisions. Phys. Rev. Lett. 1993, 70, 1771-1774.

(2) Santarelli, G.; Laurent, Ph.; Lemonde, P.; Clairon, A.; Mann, A.G.; Chang, S.; Luiten, A.N.; Salomon, C. Quantum Projection Noise in an Atomic Fountain: A High Stability Cesium Frequency Standard. Phys. Rev. Lett. 1999, 82, 4619-4622.
(3) Fertig, C.; Gibble, K. Measurement and Cancellation of the Cold Collision Frequency Shift in an ${ }^{87} \mathrm{Rb}$ Fountain Clock. Phys. Rev. Lett. 2000, 85, 1622-1625.

(4) Hardman, K.S.; Kuhn, C.C.N.; McDonald, G.D.; Debs, J.E.; Bennetts, S.; Close, J.D.; Robins, N.P. Role of Source Coherence in Atom Interferometery. Phys. Rev. A 2014, 89, 023626.

(5) Peters, A.; Chung, K.Y.; Chu, S. Measurement of Gravitational Acceleration by Dropping Atoms. Nature 1999, 400, 849-852.

(6) Gustavson, T.L.; Bouyer, P.; Kasevich, M.A. Precision Rotation Measurements with an Atom Interferometer Gyroscope. Phys. Rev. Lett. 1997, 78, 2046-2049.

(7) Brennen, G.K.; Caves, C.M.; Jessen, P.S.; Deutsch, I.H. Quantum Logic Gates in Optical Lattices. Phys. Rev. Lett. 1999, 82, 1060-1063.

(8) DeMille, D. Quantum Computation with Trapped Polar Molecules. Phys. Rev. Lett. 2002, 88, 067901.

(9) Monroe, C. Quantum Information Processing with Atoms and Photons. Nature 2002, 416, 238-246.

(10) Duan, L.M.; Lukin, M.D.; Cirac, J.I.; Zoller, P. Long-distance Quantum Communication with Atomic Ensembles and Linear Optics. Nature 2001, 414, 413-418.

(11) Anderson, M.H.; Ensher, J.R.; Matthews, M.R.; Wieman, C.E.; Cornell, E.A. Observation of Bose-Einstein Condensation in a Dilute Atomic Vapor. Science 1995, 269, 198-201.

(12) Davis, K.B.; Mewes, M.O.; Andrews, M.R.; van Druten, N.J.; Durfee, D.S.; Kurn, D.M.; Ketterle, W. Bose-Einstein Condensation in a Gas of Sodium Atoms. Phys. Rev. Lett. 1995, 75, 3969-3973.

(13) Raab, E.L.; Prentiss, M.; Cable, A.; Chu, S.; Pritchard, D.E. Trapping of Neutral Sodium Atoms with Radiation Pressure. Phys. Rev. Lett. 1987, 59, 2631-2634.

(14) Lett, P.D.; Watts, R.N.; Westbrook, C.I.; Phillips, W.D.; Gould, P.L.; Metcalf, H.J. Observation of Atoms Laser Cooled below the Doppler Limit. Phys. Rev. Lett. 1988, 61, 169-172.

(15) Hemmerich, A.; Weidemüller, M.; Esslinger, T.; Zimmermann, C.; Hänsch, T. Trapping Atoms in a Dark Optical Lattice. Phys. Rev. Lett. 1995, 75, 37-40.

(16) Boiron, D.; Michaud, A.; Lemonde, P.; Castin, Y.; Salomon, C.; Weyers, S.; Szymaniec, K.; Cognet, L.; Clairon, A. Laser Cooling of Cesium Atoms in Gray Optical Molasses Down to $1.1 \mu \mathrm{k}$. Phys. Rev. A 1996, 53, R3734-R3737.

(17) Kastberg, A.; Phillips, W.D.; Rolston, S.L.; Spreeuw, R.J.C.; Jessen, P.S. Adiabatic Cooling of Cesium to $700 \mathrm{nk}$ in an Optical Lattice. Phys. Rev. Lett. 1995, 74, 1542-1545.

(18) Kerman, A.J.; Vuletić, V.; Chin, C.; Chu, S. Beyond Optical Molasses: 3D Raman Sideband Cooling of Atomic Cesium to High Phase-space Density. Phys. Rev. Lett. 2000, 84, 439-442.

(19) Gröbner, M.; Weinmann, P.; Kirilov, E.; Nägerl, H.-C. Degenerate Raman Sideband Cooling of ${ }^{39}$ K. Phys. Rev. A 2017, 95, 033412.

(20) Li, X.; Corcovilos, T.A.; Wang, Y.; Weiss, D.S. 3D Projection Sideband Cooling. Phys. Rev. Lett. 2012, 108, 103001.

(21) Salomon, G.; Fouch, L.; Wang, P.; Aspect, A.; Bouyer, P.; Bourdel, T. Gray-molasses Cooling of $39 \mathrm{k}$ to a High 
Phase-space Density. EPL (Europhysics Letters) 2013, 104, 63002.

(22) Stellmer, S.; Tey, M.K.; Huang, B.; Grimm, R.; Schreck, F. Bose-Einstein Condensation of Strontium. Phys. Rev. Lett. 2009, 103, 200401.

(23) Takasu, Y.; Honda, K.; Komori, K.; Kuwamoto, T.; Kumakura, M.; Takahashi, Y.; Yabuzaki, T. High-density Trapping of Cold Ytterbium Atoms by an Optical Dipole Force. Phys. Rev. Lett. 2003, 90, 023003.

(24) Stellmer, S.; Pasquiou, B.; Grimm, R.; Schreck, F. Laser Cooling to Quantum Degeneracy. Phys. Rev. Lett. 2013, 110, 263003.

(25) Grimm, R.; Weidemüller, M.; Ovchinnikov, Y.B. Optical Dipole Traps for Neutral Atoms. Advances in Atomic, Molecular, and Optical Physics 2000, 42, 95-170.

(26) Cohen-Tannoudji, C.; Dupont-Roc, J. Experimental Study of Zeeman Light Shifts in Weak Magnetic Fields. Phys. Rev. A 1972, 5, 968-984.

(27) Dalibard, J.; Cohen-Tannoudji, C. Laser Cooling below the Doppler Limit by Polarization Gradients: Simple Theoretical Models. J. Opt. Soc. Am. B 1989, 6, 20232045.

(28) Castin, Y.; Berg-So/rensen, K.; Dalibard, J.;Mo/lmer, K. Two-dimensional Sisyphus Cooling. Phys. Rev. A 1994, 50, 5092-5115.
(29) White, J.D.; Scholten, R.E. Compact Diffraction Grating Laser Wavemeter with Sub-picometer Accuracy and Picowatt Sensitivity using a Webcam Imaging Sensor. Rev. Sci. Instrum. 2012, 83, 113104.

(30) Takase, K.; Stockton, J.K.; Kasevich, M.A. Highpower Pulsed-current-mode Operation of an Overdriven Tapered Amplifier. Opt. Lett. 2007, 32, 2617-2619.

(31) Rosatzin, M.; Suter, D.; Mlynek, J. Light-shift-induced Spin Echoes in a J=1/2 Atomic Ground State. Phys. Rev. A 1990, 42, 1839-1841.

(32) Park, C.Y.; Noh, H.; Lee, C.M.; Cho, D. Measurement of the Zeeman-like ac Stark Shift. Phys. Rev. A 2001, 63, 032512.

(33) Wood, A.A.; Turner, L.D.; Anderson, R.P. Measurement and Extinction of Vector Light Shifts Using Interferometry of Spinor Condensates. Phys. Rev. A 2016, 94, 052503.

(34) Brzozowski, T.M.; Maczynska, M.; Zawada, M.; Zachorowski, J.; Gawlik, W. Time-of-Flight Measurement of the Temperature of Cold Atoms for Short Trap-Probe Beam Distances. J. Opt. B: Quantum Semiclass. Opt. 2002, 4, 62-66.

(35) Lukman Winoto, S.; DePue, M.T.; Bramall, N.E.; Weiss, D.S. Laser Cooling at High Density in Deep Far-detuned Optical Lattices. Phys. Rev. A 1999, 59, R19-R22. 